\title{
The Development Track and Influencing Factors of Reading Ability of Chinese Elementary English Learners
}

\author{
Xiaohui Fu, Keyi Lou, Zhiqi Zhu, Liu Yang \\ East China University of Science and Technology, Shanghai, China \\ Email: fxh2573229703@163.com
}

How to cite this paper: Fu, X.H., Lou, K.Y., Zhu, Z.Q. and Yang, L. (2021) The Development Track and Influencing Factors of Reading Ability of Chinese Elementary English Learners. Open Access Library Journal, 8: e7289.

https://doi.org/10.4236/oalib.1107289

Received: March 7, 2021

Accepted: April 26, 2021

Published: April 29, 2021

Copyright $\odot 2021$ by author(s) and Open Access Library Inc.

This work is licensed under the Creative Commons Attribution International License (CC BY 4.0).

http://creativecommons.org/licenses/by/4.0/

\begin{abstract}
In order to improve inefficient English teaching methods in primary and secondary schools, this paper explores the influence of phonological awareness, syntactic awareness, morphemic awareness and pragmatic awareness on the reading ability of Chinese elementary English learners and provides improvement of teaching methods and concepts for student's different stages.
\end{abstract}

\section{Subject Areas}

Language Education

\section{Keywords}

Phonological Awareness, Syntactic Awareness, Morpheme Awareness, Pragmatic Awareness, Elementary English Learners

\section{1. 引言}

低阶英语学习者是指处在最初学习阶段(主要是中小学阶段), 元语言意 识仍在发展过程中, 尚未达到最终状态的学习者人群。作为一个复杂的心理 语言过程, 低阶英语学习者阅读能力的发展受到诸多因素的制约。据《2019 中小学英语阅读教学发展分析报告》, $61.11 \%$ 的老师认为自己的阅读教学课 效果一般, 53.68\%的学生一周内从未阅读任何英语绘本。中小学英语教学的 效率不理想, 一个重要原因是我们长期以来关注的是语言知识, 而不是中国 英语学习者早期语言能力的内部构成, 以及语言能力的跨语言影响和内部机 制。探索英语读写能力习得和发展的内在机制, 不仅有助于研究第二语言学 习的基本规律, 也有助于促进低阶英语学习者第二语言的习得和发展, 对促 进我国英语教学具有重要意义。 
阅读理解是一个复杂的社会心理过程, 它的成败受认知、语言、非语言 技能等多种因素的复杂组合影响。Matthew Davids 指出, 学习者提取大脑中 词汇的速度越快, 词汇的准确率越高, 语音、字形、词素和句法意识等元语 言意识越强, 其阅读理解能力越好。

\section{2. 语音意识}

我们一般认为英语语音意识由音节意识、尾韵意识和音域意识三部分组 成。中英文的音节差异较大, 中文单字即成一个音节, 但是英文单词多为多 音节词。例如: 当学习者可以意识到 lollipop 中分为 lo、1li、pop 三个音节, 我们就认为其具有了一定的音节意识。通过不断的替换首字母, 比如让学生 替换 cat 的首字母组成新单词, 统计可以组合的单词数量来测试学生的尾韵意 识。语音意识也可以被当成一种辨音, 即识别一种语言中声音组合的结构, 例如词、音节、音首、韵尾、音素等。认知来看, 语音意识是对语音表象的 解码过程, 低阶英语学习者可以通过语音意识来解码习得的语言, 因此语音 意识也成为低阶英语学习者语感和写作的基础。

英语语音意识的结构中存在三种表征形式, 依次为音节意识、首尾音意 识和音位意识 $[1]$ 。音节意识中的押韵意识、音位意识中的首音意识和末音分 割意识是低阶英语学习者辨别词汇差异, 准确联系音与形的重要基础。押韵 意识意味着学习者能从听到的单词中选择出与其他不押韵的词(听到 clown/crown/flower/lower 辨别出那一个词不押韵 ow/au/)。首音意识意味着学 习者从听到的单词中选择出与其他在开始声音上不同的一个词 (听到 number/nut/note/link 中, 辨别哪一个词开头听起来不一样)。末音分割意味 着学习者从听到的单词中找出一个与另外单词在最后一个声音上的不同(听 到 spice/trice/pice/nose 中, 辨别出哪一个词结尾听起来不一样)。

低阶英语学习者的音位意识单词阅读和拼写的显著预测作用得到李蓓蕾 等的验证[2]。低阶英语学习者的音节意识对其英语单词拼写的独立预测作用 也已得到韦晓保[3]的证实, 其结论也符合 Ziegler \& Goswami (2005)精神语言 学的微粒子理论。

拼写过程是低阶英语学习者用特定的符号表达和输出语音的过程, 是将 心理表征下的书面语言外化为社会规定的标记形式。语音意识有助于低阶英 语学习者准确、流利地识别单词, 通过应用语音对应知识对未知单词进行解 码。研究表明, 学习者只有成功解码语音, 才能完成正确的拼写过程, 如: sow/seu/播种和 sow/sau/母猪, 根据语音将单词区别记忆, 学习者才可以在相 关语境快速识别, 语音意识对语境中推测词汇含义具有显著作用 (Caravolas 2001)。

与中文文本不同, 英文文本是按字母排列的, 当字母和声音之间有对应 关系时, 低阶英语学习者才能够准确、流利地完成词汇的识别任务。如 trail 和 trial 形相近但语素不同, 学习者根据语音差异可以快速辨别和确认这两个 不同单词。若依赖汉语的以形记意, 则容易混淆二者含义。语音意识和英语 读写能力联系密切且相互作用, 汉语低阶英语学习者早期就应该有意识地利 
用语音意识加工策略读写英语词汇。因此, 在低阶英语学习者英语学习中, 教师在强调形的记忆和背诵时, 要时刻结合所对应的音, 培养有效语音解码 的能力(辨别词汇差异), 对于其书面读写能力的发展具有重要意义。

\section{3. 句法意识}

句法意识是指学习者对语言句法方面的自觉推理, 以及对语法规则使用 的自觉控制(Gombert 1992), 侧重于从结构角度建立理解。句法意识一直是学 习者阅读理解表现的预测因素(Muter et al. 2004), 与单词阅读和阅读理解有密 切的关系。

对二语学习者的研究中, Rabia \& Siegel (2002)发现句法意识与阅读理解 相关系数为 0.57 。从已有研究来看, 测量句法意识的方式主要包括了完形填 空、词序调整以及语法错误更正等。实证研究发现, 阅读流畅性涉及拼写、 发音、语素、句法等阅读相关认知技能的加工整合, 准确和完整的认知过程 是阅读理解能力的基础阶段(Kauda \& Guthrie 2008)。流畅性干预研究发现, 学生在反复阅读过程中学会有效地使用语素、语义和句法线索, 可以进一步 提高阅读的流畅性和理解能力(Kuhn \& Stahl 2003)。

句法意识对不同年龄段的阅读理解能力的影响存在差异，多项研究阐明 了这一结论。Demont 与 Gombert 等人对 20 多名来自同一地区的儿童进行了 时长 4 年的跟踪研究, 在阅读学习的最初阶段进行了大量调查实验, 探索并 总结了句法意识与阅读能力的关系。他们对数据按照顺序逐步回归分析后发 现，剔除智能和词汇水平等外部变量后，儿童的语音意识能够预测其后的编 码能力, 句法意识与阅读理解的预测一致。Kate 进行的另一项研究表明, 句 法意识和阅读理解能力的关系取决于词汇量、语法知识以及记忆这三个因素。

Orly 与 Linda 针对低阶英语二语学习者(ESL)开展了研究, 并且通过与英 语为母语(L1)的低阶英语学习者进行对比, 来分析造成该差异的深层原因。 ESL 在一年级时表现出次于 L1 的语言能力, 而在三年级时, 除句法意识之外, ESL 与 L1 两个群体的低阶英语学习者在各方面表现都相当接近, ESL 达到了 与 L1 几乎同等的阅读能力。在此之后, 句法意识作为最突出的因素影响着以 英语为第二语言的学习者阅读能力的发展。

同时, 香港对 136 名 11 岁中文学生进行的一项研究表明, 严格控制其他 影响因素时, 句法意识对阅读能力有着独特且强有力的影响, 这一点学生进 行完形填空时体现得尤为突出。Kouider 与 H. Brian 进行的一项研究则从另一 个方向验证了该结论, 句法意识不强的五年级学生, 对应着较低的阅读流畅 度和阅读理解能力。其余研究表明, 句法意识则是预测初三学生阅读理解的 重要因素, 同等情况下句法意识对阅读理解的贡献大于语音意识 [4]。

句法意识对阅读理解能力的影响在学习者不同年龄段的差异意味着, 小 学低年级阅读理解能力主要与语音意识联系密切, 而中高年级时则与句法意 识更加密切联系。因此, 对于中高年纪的学习者阅读理解能力的培养, 教师 就应该侧重于语法规则的教学, 以及倡导从结构角度建立整体理解, 而不是 将文本拆解成词单个突破。除此而外，教师应该强调反复阅读来进一步促进 
其阅读的流畅性和理解能力。

\section{4. 语素意识}

语素意识对中国低阶英语学习者的阅读能力起着重要作用, 是个体识别、 思考和运用语言形式和结构的能力, 意味着学习者可以对词的语素结构做出 及时的反应和认识。目前, 尚无法完全明确语素意识与阅读能力关系。一些 研究结果表明, 语素意识是语音意识的一部分, 在语音意识之外不能单独影 响外语阅读能力; 儿童 6 7 岁时语素意识便出现, 且随着儿童年龄的递增, 不断发展。

近年来, 研究者发现语素和语音意识间存在一定联系, 随着年龄的增长, 语音意识对低阶英语学习者阅读能力的影响逐渐减弱, 而语素意识的作用逐 渐增强(Singson 2000)。在一项针对二至五年级低阶英语学习者的研究中, Kirby 测量了学习者读错误词时反应时间, 例如单独看到 surfecelactian 时, 学习者会根据熟悉的 faceltion 组合立刻辨别出错误。篇章阅读时, 发现错误 词的反应时间增加但少于辨别篇章句子中语法错误和逻辑错误所用时间。结 果表明, 语素意识可以显著预测错误词的阅读和阅读理解 [5]。

为了排除语音意识对阅读能力的影响, 单独研究语素意识的影响, 我们 采取问卷调查的形式, 对上海市华东理工大学附属小学四年级 254 名学生的 阅读能力进行调查, 共收集 246 份有效问卷。问卷分三个步骤, 首先测定学 生的屈折语素意识对英语阅读能力的影响, 选用十个一般现在时的句子, 让 学生将其修改为一般过去时的句子。第二项测定学生的复合语素意识, 通过 提供十组相互对称的词根, 例如 motor + cycle $=$ motorcycle (摩托车), 供学生 进行匹配选择。第三项测定学生的派生语素意识, 通过提供十组相互对称的 词缀和词根, 例如 mul + berry = mulberry (桑树), 供学生进行匹配选择。结 果表明, 语素意识与低阶英语学习者的阅读能力联系密切, 屈折语素意识贡 献了 7.4\%的词汇阅读变异, 复合语素意识贡献了 $18.3 \%$ 的新造词和临时造词, 派生语素意识贡献了 $13.5 \%$ 。

研究人员调查了三种类型的英语语素词, 结果发现只有屈折词、合成语 素对阅读有贡献，派生词汇意识的预测作用没有达到显着水平[6]。通过对其 他文献的研究发现, 对于低阶英语学习者来说, 派生词汇意识能力通常只在 小学阶段中高年级发展。因此, 作为汉语的母语英语学习者, 可以理解由于 缺乏适当的环境, 衍生词意识的发展缓慢, 甚至滞后是可以理解的。

在英语教学中, 通过注重语法, 可以有效地加强英语学习者的屈曲意识, 表现出与英语阅读能力的较高相关性; 合成词语素意识的发展可能与汉语的 母语环境有关, 其中大部分是合成词, 用英语学习汉语很容易迁移汉语合成 词语素意识的知识结构, 提高了英语单词的解码和理解能力。

\section{5. 语用意识}

语用意识指学生综合的语言运用意识, 需要学习者理解语言背后的深层 含义。如: 对话中未说出口的规则和惯例。从语言角度来看, 语用意识是对 
话者在话语过程中实施和理解社会和体面行为所使用的知识类型。从心理学 角度看, 语用意识是处理语言信息的内在能力。

学习者常常会混淆语言能力和语用能力, 甚至认为语言能力就等于语用 能力。这意味着听、说、读、写能力都很优秀的学习者依然可能在特定的社 会环境下产生符合交际需求的错误表达和文化误解, 例如学习者和信仰伊斯 兰教的人交流时就需要谨慎使用 God一词。在英语阅读的过程中，学习者不 局限词汇习得和课文理解, 主动了解相关历史、文化、政治经济等背景知识 产生的影响, 就能在更宏观的层面上理解文章主题。之后接触到相似背景的 文章时，也才能够更快的把握文章主题。

何周春和龚彦知[7]采用 Bordovi-Harlig 和 Dornyei (1998)设计的问卷和测 试题, 让 126 名小学生、123 名初中生、131 名高中生参与了测试。研究表明, 低阶英语学习者的语用意识培养总体上处于较低阶, 在汉语中更多地运用语 用学原则, 或者判断英语在语言上是否有误, 且对语用错误的判断更多依赖 于直觉。低阶英语学习者的阶段性特征是导致语用意识发展阶段性差异的重 要原因, 与许多因素有关, 如母语语音、语言环境、学习者个体发展等。

受教育程度低的学生能够理解接近高水平但在普通术语和言语行为上距 离较远的学生的谈话含义, 这表明在每个学习阶段都应鼓励发展不同的语言 能力, 并加强他们的实用主义意识(Rose, 1994)。对学生学习某一种语言的英 语水平进行评估的结果很难推广到其他语言技能或学生学习外语的整体语用 能力。要提高中国英语学习者的语用能力, 就必须改进英语教学的内容和模 式, 充分发挥教学在语用能力培养中的作用。

实用主义为主的教学效果最好, 建议教师在教学过程中明确语用学的相 关知识。在语用能力验证领域, 学生语用能力的不平衡发展要求语用能力验 证充分重视文件内容的真实性。Bardovi-Harlig (1999)和赵左(2009)认为, 特 定的语法知识对于相关的语用知识来说更具有可预测性, 故应该深入研究某 些特定语法能力和某些特定语用能力之间的关系, 并且重点研究英语情态动 词语用能力的可预测性。

\section{6. 结语}

根据不同阶段语音、句法、语素和语用意识与低阶学习者英语阅读能力 的联系, 教师需要合理地安排教学任务。小学阶段初期, 语音意识和英语阅 读能力的显著相关意味着教师需要更关注 “音 - 形” 对应关系的外显教学。 语音意识在后期对阅读能力的影响逐渐降低, 语素意识和句法意识逐渐增强, 故小学阶段的中后期应注重语法的教学和结构角度的整体理解。进入中学阶 段后, 教师则应该重视培养学生的语用意识, 并重点讲解语用学的相关知识。

需要指出的是, 目前本文中提到研究结论大多针对英语为母语的低阶英 语学习者进行推断的。因此, 以汉语为母语的低阶英语学习者要过渡到以英 语为第二语的过程还需要进一步的研究。除此而外, 此次元语音意识对阅读 能力的影响因素的结论是在非自然语境下调查的, 因而中国低阶英语学习者 的元语音意识发展的特征与规律还需通过访谈、田野调查等方法获得大量原 始数据后分析, 才可得以更为充分的体现。 


\section{Conflicts of Interest}

The authors declare no conflicts of interest regarding the publication of this paper.

\section{References}

[1] 徐芬. 低阶英语学习者汉语和英语语音意识的发展特点及其相互关系 [D]: [博士 学位论文]. 杭州: 浙江大学, 2002.

[2] 李蓓蕾, 陶沙, 董奇. 英语语音意识在汉语低阶英语学习者英语单词阅读及拼写 中的作用[J]．心理发展与教育, 2011, 27(4): 388-393.

[3] 韦晓保. 语音和韵律意识在汉语低阶英语学习者英语单词拼写中的作用[J]. 解放 军外国语学院学报, 2017, 40(4): 79-86+160.

[4] Kouider, M. and Brian Thompson, H. (2006) How Problems of Reading Fluency and Comprehension and Related to Difficulties in Syntactic Awareness Skills among Fifth Grader. Literacy Research and Instruction, 46, 73-94. https://doi.org/10.1080/19388070609558461

[5] 张方蕾. 小学生语素意识与阅读理解的关系研究 [D]: [硕士学位论文]. 西安: 陕 西师范大学.

[6] 常云. 汉语儿童英语语素意识与阅读学习的关系 [J]. 内蒙古师范大学学报, 2010(4): 103-107.

[7] 何周春, 龚彦知. 中国英语学习者的语言意识发展研究一一以语用意识与语法意 识为视角 [J]. 现代外语, 2013, 36(3): 278-285+330.

\section{Appendix (Abstract and Keywords in Chinese)}

\section{中国低阶英语学习者阅读能力发展轨迹及影响因素}

摘要: 针对中小学英语教学效率不理想的问题, 本文探讨整合了语音意 识、句法意识、语素意识和语用意识对中国低阶英语学习者阅读能力的影响 并且针对不同阶段提供了教学方法和理念的改进。

关键词：语音意识, 句法意识, 语素意识, 语用意识, 低阶英语学习者 